\title{
Primeiro registro de Tomoplagia reimoseri Hendel e Xanthaciura mallochi Aczél (Diptera: Tephritidae) para o Estado de Santa Catarina, Brasil
}

\author{
First records of Tomoplagia reimoseri Hendel e Xanthaciura mallochi Aczél (Diptera: Tephritidae) \\ for Santa Catarina state, Brazil
}

\author{
Sandra Mara Sabedot ${ }^{\mathrm{I}}$ Flávio Roberto Mello Garcia ${ }^{\mathrm{I}, \mathrm{II}}$ Gilson Marcos Bogus \\ Marcoandré Savaris ${ }^{\mathrm{I}}$
}

\begin{abstract}
O presente trabalho tem como objetivo levantar as espécies de tefritídeos endófagos associados a capítulos de asteráceas em Chapecó, Santa Catarina. Para tanto, foram efetuadas coletas quinzenais no município de Chapecó, Santa Catarina, durante o período de setembro de 2005 a setembro de 2006, concentradas na primavera e no verão. Os capítulos foram destacados diretamente das plantas, no campo, e acondicionados em sacos plásticos. Este trabalho constitui o primeiro registro de Tomoplagia reimoseri Hendel $e$ Xanthaciura mallochi Aczél coletados em capítulos de Asteraceae no município de Chapecó, elevando para 72 espécies de tefritídeos registradas para o referido Estado.
\end{abstract}

Palavras-chave: insetos endófagos, Asteraceae, Tomoplagia, Xanthaciura.

\section{ABSTRACT}

The present research aimed to inventory the species of endophagous tephritids associated to Asteraceae capitula in Chapecó, Santa Catarina. Samples of capitula were collected every two weeks in Chapecó, from september 2005 to september 2006, concentrating efforts on spring and summer. The capitula were collected directly from the plants and conditioned in plastic bags. This research constitutes the first register of Tomoplagia reimoseri Hendel and Xanthaciura mallochi Aczél specimens collected from Asteraceae capitula in Chapecó, elevating to 72 the number of tephritid species registered to the State of Santa Catarina.

Key words: endofages insects, Asteraceae, Tomoplagia, Xanthaciura.
No Estado de Santa Catarina, existem poucos dados de distribuição geográfica, bioecologia e levantamento de tefritídeos endófagos. Os dados que existem são de pesquisas realizadas nas cidades de Mafra, Matos Costa, Lages, Bom Jardim da Serra, Pericó, São Joaquim e Santa Cecília (PRADO et al. 2002), sendo até o momento registradas 34 espécies de tefritídeos endófagos (GARCIA et al. 2002).

Diptera possui importância diversificada, principalmente na área agrícola, sendo que algumas espécies são polinizadoras e desempenham importante papel junto à natureza. A família Tephritidae abrange as moscas frugívoras, relacionadas principalmente com plantas de importância agrícola (GARCIA 2002), sendo bastante diversificada e abundante de endófagos de capítulos de Asteraceae no Brasil.

A família de plantas hospedeiras com o maior número de espécies de Tephritidae associadas no mundo é a Asteraceae (ZWÖLFER, 1987; FOOTE et al. 1993). A associação com as asteráceas é antiga e parece ter sido uma das principais razões da diversificação dos Tephritidae (ZWÖLFER, 1987). A grande diversidade e a distribuição cosmopolita da subfamília Tephritinae sugerem a ocorrência de uma grande dispersão, após a colonização das asteráceas. No Brasil, os endófagos de capítulos de asteráceas pertencem, principalmente, a três famílias de dípteros (Tephritidae, Cecidomyiidae e Agromyzidae) e quatro

IUniversidade Comunitária Regional de Chapecó (UNOCHAPECÓ), Programa de Mestrado em Ciências Ambientais. Laboratório de Entomologia, CP 747, 89809-0000, Chapecó, SC, Brasil. E-mail: sandrams@unochapeco.edu.br.*Autor para correspondência.

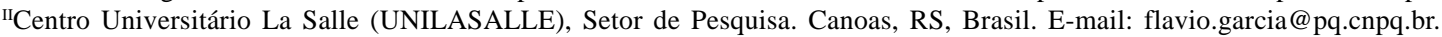


de microlepidópteros (Tortricidae, Pterophoridae, Blastobasidae, Pyralidae, Gelechiidae), totalizando mais de 120 espécies (LEWINSOHN, 1988; 1991).

Sendo assim, o presente trabalho tem como objetivo levantar as espécies de tefritídeos endófagos associados a capítulos de asteráceas em Chapecó, Santa Catarina.

As coletas foram efetuadas quinzenalmente no referido município (2709'S; '52 ${ }^{\circ} 61 \mathrm{~W}$ ), durante o período de setembro de 2005 a setembro de 2006, concentradas na primavera e no verão, devido ao período de floração da maioria das espécies de asteráceas, na região oeste de Santa Catarina. Quando as plantas diferiam em seu estado fenológico, incluíram-se plantas com diferentes estágios de florescimentos, excluindo-se as que se encontrava em pré-antese ou que estavam com seus aquênios inteiramente maduros. Foram escolhidas preferencialmente espécies herbáceas ou arbustivas por permitirem maior eficiência na coleta, tornando-se possível a coleta de grande número de capítulos. A maioria das espécies de asteráceas amostradas é nativa do Brasil, com exceções para as espécies: Chromolaena margaritensis (eupatório), Chromolaena laevigata (cambará-falso), Conyza canadensis (buva), Chrysolaena flexuosa (assapeixe), Tagetes patula (tagetes), Bidens pilosa (picão-preto) e Baccharis trinervis (assapeixe-branco). Os capítulos foram destacados diretamente das plantas, no campo, e acondicionados em sacos plásticos, devidamente identificados.

Para cada espécie de asterácea, foram feitas exsicatas para confirmação da identificação. Todas foram inicialmente separadas em morfoespécies e posteriormente pré-identificadas, com o auxílio de bibliografia. As exsicatas foram enviadas para o Dr. Nelson Matzenbacher para identificação e também confirmadas por meio das exsicatas de referência do Laboratório de Interação Insetos-Plantas da Universidade Estadual de Campinas (UNICAMP), e as exsicatas duplicatas encontram-se depositadas no Herbário da Universidade Comunitária Regional de Chapecó(UNOCHAPECÓ).

Os capítulos foram acondicionados no laboratório de Entomologia da UNOCHAPECÓ, onde eram mantidos em potes plásticos, sendo inspecionados diariamente para a retirada dos insetos adultos. Os insetos foram fixados e montados em alfinetes entomológicos ou acondicionados em cápsulas de gelatina.

Os tefritídeos foram separados em morfoespécies, sendo que a identificação em termos de gênero foi baseada em FOOTE (1980) e espécies de Xanthaciura em ACZÉL (1949). Posteriormente eles foram comparados com a coleção de tefritídeos endófagos do Laboratório de Interação Inseto-planta da UNICAMP. Os espécimes de Tephritidae estão depositados na coleção entomológica do Laboratório de Entomologia da UNOCHAPECÓ.

Foram registrados no total 16.472 tefritídeos, sendo destes 1.555 indivíduos de Tomoplagia e 860 de Xanthaciura, os demais pertencem aos gêneros Cecidochares, Dictyotrypeta, Dyseuaresta, Dioxyna, Neomyopites, Plaumannymia, Trupanea e Trypanaresta.

Três espécies de Tomoplagia foram identificadas: Tomoplagia incompleta Williston, Tomoplagia biseriata Loew e Tomoplagia reimoseri Hendel. Entre estas, Tomoplagia reimoseri é registrada pela primeira vez no Estado de Santa Catarina.

O gênero neotropical Tomoplagia é um dos mais abundantes entre os tefritídeos que se desenvolvem em capítulos de compostas no Brasil, sendo composto por aproximadamente 47 espécies, das quais em torno de 25 ocorrem no Brasil (YOTOKO et al., 2005; NORRBOM et al., 1998) e possui como principais hospedeiros plantas da tribo Vernonieae. Das duas espécies que ocorrem nos Estados Unidos, Tomoplagia obliqua Say está registrada para, pelo menos, sete espécies de Vernonieae, e Tomoplagia cressoni Aczél para Trixis californica (solidonia) Kellog e Perezia microcephala (DC.) A. Gray (Mutisieae) (FOOTE et al. (1993). GARCIA et al. (2002) relata a ocorrência de sete espécies de Tomoplagia para Santa Catarina: Tomoplagia biseriata (Loew, 1873), Tomoplagia costalimai (Aczél, 1955), Tomoplagia fibrigi (Hendel, 1914), Tomoplagia formosa (Aczél, 1955), Tomoplagia incompleta (Williston, 1896), Tomoplagia minuta (Hering, 1938) e Tomoplagia vernoniae (Hering, 1938).

As principais plantas hospedeiras da maioria das espécies de Tomoplagia pertencem à tribo Vernonieae, com exceção de três espécies, $\boldsymbol{T}$. costalimai Aczél, as quais estão associadas com Trixis sp. (Mutiseae), T. trivittata Lutz and Lima, associada à Gochnatia sp. (cambará) (Mutiseae), e T. biseriata Loew com Calea sp. (erva-rasteira) (Heliantheae). Plantas das tribos Mutiseae e Heliantheae são ocasionalmente associadas por algumas espécies de Tomoplagia (PRADO et al., 2002)

Tomoplagia reimoseri, com 1.039 indivíduos, teve registro em $\boldsymbol{V}$. phosphorica (assapeixebranco) e C. pedunculosa (eupátorio), sendo consideradas hospedeiras principais. Nesse estudo, a espécie foi coletada em ambiente rural e urbano, principalmente em área rural, nas proximidades de estradas. 
Xanthaciura é um gênero neotropical e neártico, com 17 espécies descritas (NORRBOM et al., 1998). Ele ocorre amplamente nas regiões Nordeste, Central, Sudeste e Sul do Brasil, e está associado às tribos Heliantheae e Eupatorieae e ocasionalmente em plantas da Tribo Heliantheae (PRADO et al., 2002). Para Santa Catarina, estão registradas quatro espécies: $\boldsymbol{X}$. biocellata (Thompson, 1869), X. chrysura erosa (Thompson, 1869), X. quadrisetosa (Hendel, 1914) e X. unipuncta (Malloch, 1933) (GARCIA et al. 2002).

Houve registro de $\boldsymbol{X}$. mallochi em Chromolaena laevigata, a qual ocorreu ocasionalmente. PRADO et al. (2002) obteve $\boldsymbol{X}$. mallochi em Chromolaena maximilianii (mata-pasto) (Eupatorieae), indicando que a espécie está primariamente associada com a referida tribo, com registro apenas para o Estado de Minas Gerais.

São adicionadas duas espécies à lista de GARCIA et al. (2002) e GARCIA (2002), passando a ser conhecidas 72 espécies em Santa Catarina.

\section{AGRADECIMENTOS}

Agradecimentos à UNOCHAPECÓ, pelo auxílio com a bolsa de pesquisa ao Dr. Allen L. Norrbom, do Systematic Entomology Laboratory (USDA), e ao Prof. Dr. Paulo Inácio Prado, do Departamento de Ecologia do Instituto Biociências da Universidade de São Paulo, pela contribuição na identificação de algumas espécies de tefritídeos, e ao Dr. Nelson Matzenbacher, pela identificação das asteráceas.

\section{REFERÊNCIAS}

ACZÉL , M.L. A revision of The genus Xanthaciura Hendel (Trypetidae, Diptera) based on argentine species. Acta Zoologia Lilloana, v.8, p.111-146, 1949.

FOOTE, R.H. Fruit fly genera South of the United States. Washington: U.S. Governement, 1980. 79p. (Technical Bulletin, 1600).
FOOTE, RH. et al. Handbook of the fruit flies (Diptera: Tephritidae) of America and North of Mexico. Ithaca: Comstock, 1993.

GARCIA, F.R.M. Ocorrência de moscas-das-frutas (Diptera, Tephritidae) de Santa Catarina, Brasil. Acta Ambiental Catarinense, Chapecó, v.1, n.2, p.81-83, 2002.

GARCIA, F.R.M. et al. Lista documentada das moscas-dasfrutas (Diptera, Tephritidae) de Santa Catarina, Brasil. Biociências, Porto Alegre. v.10, n.1, p.139-148, 2002.

LEWINSOHN, T.M. Composição e tamanho de faunas associadas a capítulos de compostas. 1988. Tese (Doutorado em Ecologia) - Universidade Estadual de Campinas, Campinas.

LEWINSOHN, T.M. Insects in flower heads of Asteraceae in Southeast Brazil: a tropical case study on species richness. In: PRICE, P.W. et al. Plant-animal interactions: Evolutionary ecology in tropical and temperate regions. New York: Wiley/Interscience, 1991. p.560.

NORRBOM, A.L. et al. Status of knowledge of Tephritid systematics. In: THOMPSON, F.C. Fruit Fly expert identification system and systematic information. Leiden: Backhuys Publishers, 1998.

PRADO, P.I.K.L. et al. The fauna of Tephritidae (Diptera) from capitula of asteraceae in Brazil. Proceedings of the Entomological Society of Washington. v.104, n.4. p.10071028, 2002.

YOTOKO, K.S.C. et al. Testing the trend towards specialization in herbivore-host plant associations using a molecular phylogeny of Tomoplagia (Diptera: Tephritidae). Molecular phylogenetics and evolution. v.35. p.701-711, 2005.

ZWÖLFER, H. Species richness, species packing and evolution in insect-plant systems. In: SCHULZE, E.D.; ZWÖLFER, H. Potentials and Limitations of Ecosystem Analysis. Berlin: Springer-Verlag, 1987. 